\title{
Current and Current Fluctuations in Quantum Shuttles
}

\author{
Jauho, Antti-Pekka; Flindt, Christian; Novotny, Tomas; Donarini, Andrea
}

Published in:

Physics of Fluids

Link to article, DOI:

10.1063/1.1949207

Publication date:

2005

Document Version

Publisher's PDF, also known as Version of record

Link back to DTU Orbit

Citation (APA):

Jauho, A-P., Flindt, C., Novotny, T., \& Donarini, A. (2005). Current and Current Fluctuations in Quantum Shuttles. Physics of Fluids, 17(10), 100613. https://doi.org/10.1063/1.1949207

\section{General rights}

Copyright and moral rights for the publications made accessible in the public portal are retained by the authors and/or other copyright owners and it is a condition of accessing publications that users recognise and abide by the legal requirements associated with these rights.

- Users may download and print one copy of any publication from the public portal for the purpose of private study or research.

- You may not further distribute the material or use it for any profit-making activity or commercial gain

- You may freely distribute the URL identifying the publication in the public portal

If you believe that this document breaches copyright please contact us providing details, and we will remove access to the work immediately and investigate your claim 


\title{
Current and current fluctuations in quantum shuttles
}

\author{
Antti-Pekka Jauho a) \\ NanoDTU, MIC-Department of Micro and Nanotechnology, Technical University of Denmark, \\ DTU-Building 345east, DK-2800 Kongens Lyngby, Denmark \\ Christian Flindt ${ }^{\text {b) }}$ \\ NanoDTU, MIC-Department of Micro and Nanotechnology, Technical University of Denmark, \\ DTU—Building 345east, DK-2800 Kongens Lyngby, Denmark \\ Tomáš Novotnýc) \\ NanoDTU, MIC-Department of Micro and Nanotechnology, Technical University of Denmark, \\ DTU-Building 345east, DK-2800 Kongens Lyngby, Denmark and Department of Electronic Structures, \\ Faculty of Mathematics and Physics, Charles University, Ke Karlovu 5, 12116 Prague, \\ Czech Republic \\ Andrea Donarinid) \\ NanoDTU, MIC-Department of Micro and Nanotechnology, Technical University of Denmark, \\ DTU—Building 345east, DK-2800 Kongens Lyngby, Denmark
}

(Received 11 January 2005; accepted 12 April 2005; published online 3 October 2005)

\begin{abstract}
We review the properties of electron shuttles, i.e., nanoelectromechanical devices that transport electrons one by one by utilizing a combination of electronic and mechanical degrees of freedom. We focus on the extreme quantum limit, where the mechanical motion is quantized. We introduce the main theoretical tools needed for the analysis, e.g., generalized master equations and Wigner functions, and we outline the methods how the resulting large numerical problems can be handled. Illustrative results are given for current, noise, and full counting statistics for a number of model systems. Throughout the review we focus on the physics behind the various approximations, and some simple examples are given to illustrate the theoretical concepts. We also comment on the experimental situation. (C) 2005 American Institute of Physics. [DOI: 10.1063/1.1949207]
\end{abstract}

\section{INTRODUCTION}

As advances in technology push the size of the electronic components towards the nanometer scale, the wellestablished technology of micorelectromechanical systems begins to acquire quantum features. This trend signals the birth of a new research field, nanoelectromechanical systems (NEMS). Despite of the infancy of NEMS, a large literature is already available on it, and for a broad overview the interested reader is referred to recent review papers. ${ }^{1-3}$ The review at hand has a more restricted scope: it is devoted to a theoretical analysis of a very specific NEMS device, the electron shuttle. By presenting a detailed study of such an idealized model system, we hope to be able to illustrate the basic physics and conceptual problems that need to be understood before a general theory of NEMS can be developed.

The electron shuttle, originally introduced by Gorelik et al. in $1998,{ }^{4}$ consists of a movable nanoscopic grain which is coupled via tunnel barriers to source and drain electrodes. A subtle combination of the quantum transport and the mechanical degrees of freedom play an essential role for the functionality of the device. The device can exhibit a "phase transition": when a control parameter is tuned (this could be the damping of the oscillator), at a certain threshold value the

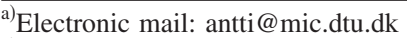

${ }^{b)}$ Electronic mail: cf@mic.dtu.dk

c)Electronic mail: andrea.donarini@physik.uni-regensburg.de

${ }^{\mathrm{d})}$ Electronic mail: novotny@fys.ku.dk
}

system enters a new transport regime, the shuttle regime, where charge is transported in an orderly fashion (i.e., essentially a fixed number of charges per mechanical oscillation cycle). (This phenomenon is particularly pronounced if the device operates in the strong Coulomb blockade regime, where only one excess charge at a time is allowed in the movable part.) In the original suggestion of Ref. 4, the motion of the movable grain was treated macroscopically, i.e., with Newton's equation of motion. In our group, we have been asking questions regarding what happens when the movable body is so light that its motion also becomes quantized. For example, does the shuttle transition persist in the quantum regime? Another interesting question concerns the interpretation of measurements of NEMS devices, possibly exhibiting shuttling. As it turns out, a measurement of the stationary IV characteristics does not always yield enough information to uniquely identify the underlying microscopic charge transport mechanism. A point in case is the $\mathrm{C}_{60}$ single-electron transistor experiment by Park et al. ${ }^{5}$ where two alternative interpretations, namely, incoherent phonon assisted tunneling $^{6-9}$ or shuttling, ${ }^{4,10}$ are plausible. The current noise provides another important characteristics, supplementary to the mean current. ${ }^{11-13}$ The Fano factor which characterizes the degree of correlation between charge transport events is a powerful diagnostic tool to distinguish between various transport mechanisms. Therefore, studies of the current noise in NEMS have become an active field of research. ${ }^{14-20}$ Based on the studies where the mechanical 
system is treated classically, one expects a giant enhancement of noise at the shuttling transition, ${ }^{18}$ and we want to investigate whether this also occurs in the quantum case. One can go even further along this line: why stop at the current noise, which is the second cumulant of the full counting statics (FCS)? Surely FCS [which equals the probability $P_{n}(t)$ of $n$ electrons being collected, say, in the right lead in the time span 0 to $t$ ] will contain even more detailed information. Consequently, FCS in mesoscopic devices has been a very active research field for some time now, and its importance and relevance have been underlined by a recent measurement of the third cumulant. ${ }^{21}$ Thus, FCS for NEMS is obviously an interesting issue. We are aware of a recent calculation for a classical, driven shuttle,${ }^{15}$ and at the end of this paper we illustrate some of our own very recent generalizations of these concepts to the quantum regime using a simple example; a full description is given elsewhere. ${ }^{22}$

We have investigated quantum shuttles in several recent papers, ${ }^{19,22-26}$ where the full technical details can be found; the purpose of the present paper is to introduce some of the basic issues to a more general reader. Several other groups have also recently studied quantum shuttles, see, e.g., Refs. 27-30. The paper is organized as follows. In Sec. II, we introduce the models of quantum shuttles employed in our work. The total Hamiltonian consisting of the "system" (both mechanical and electronic degrees of freedom of the quantum $\operatorname{dot}(\mathrm{s})$ ), the leads, and a generic heat bath is used to illustrate the derivation of a Markovian generalized master equation (GME) which is the starting point of the theoretical analysis. Along the way from the Hamiltonian to the generalized master equation we identify the necessary assumptions and point out several issues of potential importance not addressed so far within the field of NEMS.

In Sec. III, we discuss the calculation of the current and the zero-frequency component of the current noise spectrum for a NEMS device described by a Markovian GME. We also give a qualitative discussion of the required numerical calculations, which are highly nontrivial due to the large dimensions of the involved matrices. Finally, some examples of our numerical results are displayed. Section IV is devoted to an elementary discussion of the calculation of the full counting statistics, and a brief review of the experimental status.

\section{THE MODEL}

At least two model systems have been considered in the literature. Gorelik et al. ${ }^{4}$ considered a single movable quantum dot, while Armour and MacKinnon ${ }^{31}$ introduced a model of a three-dot array whose central dot is movable, see Fig. 1. Both these systems are of intrinsic interest and may find applications in real systems. The quantum system is assumed to be in the strong Coulomb blockade regime in which none or only one extra (spinless) electron in the whole system is allowed. Thus, in the one-dot case the electronic states are $|0\rangle$ and $|1\rangle$, while in the triple-dot case we have $|0\rangle,|L\rangle,|C\rangle$, and $|R\rangle$. The quantum system is coupled to two leads with a high bias applied between them. The bias is smaller than the charging energy for addition or removal of other electrons but otherwise it is the largest energy scale in the model.

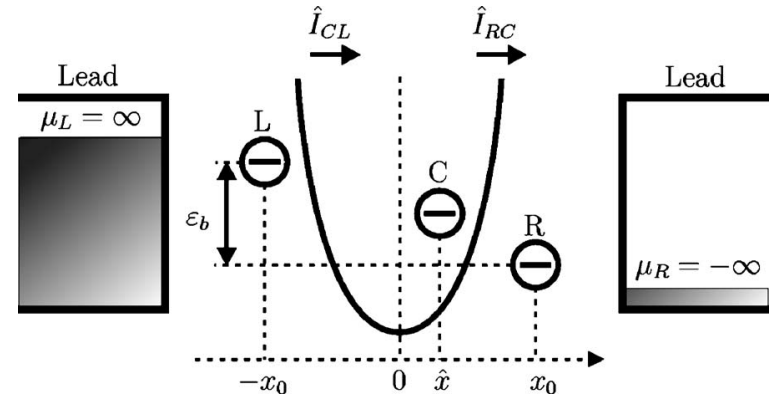

FIG. 1. Schematic picture of a three-dot system, introduced by Armour and MacKinnon (Ref. 31). The outer dots are fixed-the left one $L$ at the position $-x_{0}$ and the right one $R$ at $x_{0}$, while the central one $C$ can move (position $\hat{x}$ ) in a harmonic confining potential. It also interacts with a heat bath causing damping and thermal noise. The outer dots whose respective energy levels are dealigned by the device bias $\varepsilon_{b}$ are coupled to the full or empty electronic reservoirs (leads), respectively. The current flows within the system due to tunneling between the left and central dots and the central and right dots. (Reproduced from Ref. 25.)

The moving dot interacts with its surroundings and its dissipative dynamics is described by the interaction with a generic heat bath. The Hamiltonian has the form

$$
\hat{H}=\hat{H}_{\text {osc }}+\hat{H}_{\text {el }}+\hat{H}_{\text {el-leads }}(\hat{x})+\hat{H}_{\text {leads }}+\hat{H}_{\text {bath }}+\hat{H}_{\text {osc-bath }},
$$

where

$$
\hat{H}_{\mathrm{osc}}=\frac{\hat{p}^{2}}{2 m}+\frac{m \omega_{0}^{2} \hat{x}^{2}}{2}
$$

describes the mechanical center-of-mass motion of the central dot as a one-dimensional harmonic oscillator with mass $m$ and frequency $\omega_{0}$. We emphasize the importance of the nonlinear dependence of $\hat{H}_{\text {el-leads }}$ on the oscillator degree of freedom; the $\hat{x}$ dependence is often exponential which lies at the heart of the shuttling instability. We refer to the literature for explicit expressions for the other terms appearing in Eq. (1a). ${ }^{4,231}$ The leads are held at different electrochemical potentials $\mu_{L, R}$ whose difference gives the bias across the array [see Fig. 1]. We assume that the tunneling densities of states are independent of energy. This is necessary for the first Markov approximation, ${ }^{32}$ used later on, to hold. Further, as already implied above, we assume $\mu_{L} \rightarrow \infty, \mu_{R} \rightarrow-\infty$. These assumptions are necessary for the derivation of the Markovian dynamics of the movable system. At present, there are several open questions concerning the correct form of the appropriate non-Markovian generalized master equation for shuttle systems (also, the numerical methods are available only for Markovian systems), and calculations for quantum shuttles as a function of the external bias have not been reported, at least to the best of our knowledge. We are thus restricted to presenting results as a function of the damping, which cannot be tuned experimentally in any straightforward fashion, or, in the case of a triple-dot shuttle (see Fig. 1), as a function of the device bias, which can be tuned by gates. Thus, our calculations should be distinguished from the pioneering work of Gorelik et al., ${ }^{4}$ which studied the shuttling transition of a classical shuttle as a function of external bias. We do believe, however, that also the quantum shuttle can 


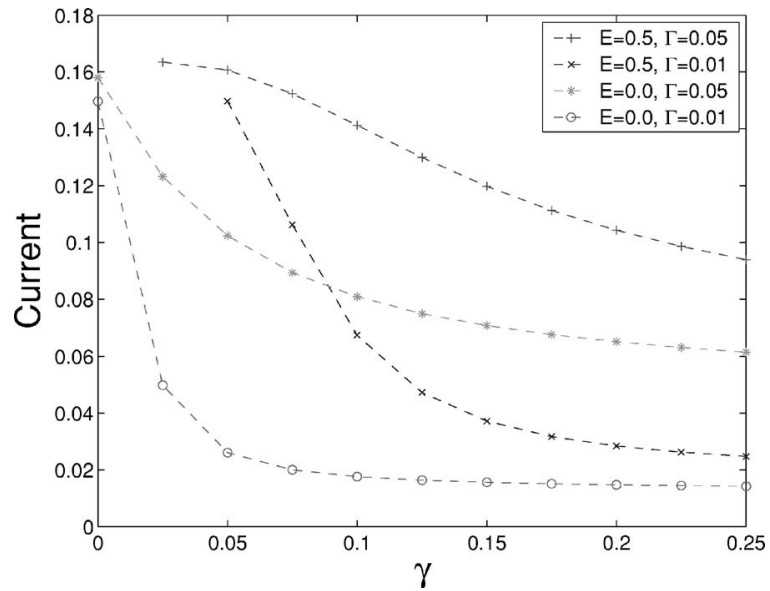

FIG. 2. I- $\gamma$ curve. The damping dependence of the stationary current through the single-dot shuttle for different transfer rates and electric fields parametrized by $d=e E / m \omega^{2}$. Their values are $d=0.5 x_{0}, \Gamma=0.05 \omega$ (pluses; corresponds to Fig. 3), $d=0.5 x_{0}, \Gamma=0.01 \omega$ (circles), $d=0.0, \Gamma=0.05 \omega$ (asterisks), $d=0.0, \Gamma=0.01 \omega$ (crosses). Other parameters are $\lambda=x_{0}, T=0$. The current is in units of $e \omega$ while $\gamma$ in $\omega$. (Reproduced from Ref. 23.)

exhibit the shuttling transition as the bias is varied. There are three pieces of indirect evidence suggesting this. (i) The quantum shuttle bears in many respects a strong analog with the classical shuttle, where the transition does take place. (ii) We can perform calculations for different values for the electric field $E$ acting on the shuttle [see Fig. 2 and Eq. (3) below], and we observe the shuttling transition (or a crossover) taking place as the electric field is increased. (iii) For a triple-dot shuttle, we observe a shuttling transition as the device bias is varied. In summary, developing a theory at finite bias for a quantum shuttle is the outstanding theoretical challenge. Finally, the heat bath consisting of an infinite set of harmonic oscillators linearly coupled to the mechanical degree of freedom is described with the Caldeira-Leggett model $^{33}$ in its Ohmic form.

\section{Generalized master equation}

For the description of the model we use the language of quantum dissipative systems. ${ }^{33}$ As the system (or "device") we take the electronic states plus the one-dimensional oscillator describing the center-of-mass motion of the central dot. The electronic leads and the heat bath interacting with the mechanical degree of freedom constitute the reservoirs. The task is to integrate out the degrees of freedom of the reservoirs to end up with an equation of motion for the system density operator. The derivation proceeds in two steps, first integrating out the leads and then the heat bath in the weak coupling limit to get the desired GME for the system density operator. We remark that the assumed additivity of the two heat baths should be proven; however, we are not aware of any such proof.

As a specific example of an end result of this procedure, we give the GME governing the system density operator in the one-dot case, written here in a Liouvillean form

$$
\dot{\rho}(t)=\mathcal{L} \rho(t)=\left(\mathcal{L}_{\text {coh }}+\mathcal{L}_{\text {driv }}+\mathcal{L}_{\text {damp }}\right) \rho(t)
$$

where the various superoperators $\mathcal{L}_{i}, i=\mathrm{coh}$, driv, damp, are defined as

$$
\begin{aligned}
\mathcal{L}_{\mathrm{coh}} \rho= & \frac{1}{i \hbar}\left[H_{\mathrm{osc}}+\varepsilon_{0} c_{0}^{\dagger} c_{0}-e E x c_{0}^{\dagger} c_{0}, \rho\right], \\
\mathcal{L}_{\text {driv }} \rho= & -\frac{\Gamma_{L}}{2}\left(c_{0} c_{0}^{\dagger} e^{-2 x / \lambda} \rho-2 c_{0}^{\dagger} e^{-x / \lambda} \rho e^{-x / \lambda} c_{0}\right. \\
& \left.+\rho e^{-2 x / \lambda} c_{0} c_{0}^{\dagger}\right)-\frac{\Gamma_{R}}{2}\left(c_{0}^{\dagger} c_{0} e^{2 x / \lambda} \rho-2 c_{0} e^{x / \lambda} \rho e^{x / \lambda} c_{0}^{\dagger}\right. \\
& \left.+\rho e^{2 x / \lambda} c_{0}^{\dagger} c_{0}\right), \\
\mathcal{L}_{\text {damp }} \rho= & -\frac{i \gamma}{2 \hbar}[x,\{p, \rho\}]-\frac{\gamma m \omega}{\hbar}(\bar{N}+1 / 2)[x,[x, \rho]] .
\end{aligned}
$$

The physical meaning of some of the terms is of particular interest. The term proportional to $E$ is due to the electrostatic force on an occupied (i.e., charged) dot. The exponential dependence of the tunneling terms is clearly visible. The damping of the oscillator cannot be described entirely satisfactorily in the Markovian limit: one cannot simultaneously achieve translational invariance, positivity of the density matrix, and relaxation towards canonical equilibrium. The form given above does not satisfy strict positivity; the quantitative consequences of this shortcoming turned out to be negligible. ${ }^{23}$ To proceed further, one considers the electronic diagonal elements $\rho_{00}(t)=\langle 0|\rho(t)| 0\rangle$ and $\rho_{11}(t)=\langle 1|\rho(t)| 1\rangle$, where $|1\rangle=c_{0}^{\dagger}|0\rangle$. These objects are still full density matrices in the phonon space and satisfy

$$
\begin{aligned}
\dot{\rho}_{00}(t)= & \frac{1}{i \hbar}\left[H_{\mathrm{osc}}, \rho_{00}(t)\right]-\frac{\Gamma_{L}}{2}\left(e^{-2 x / \lambda} \rho_{00}(t)+\rho_{00}(t) e^{-2 x / \lambda}\right) \\
& +\Gamma_{R} e^{x / \lambda} \rho_{11}(t) e^{x / \lambda}+\mathcal{L}_{\mathrm{damp}} \rho_{00}(t), \\
\dot{\rho}_{11}(t)= & \frac{1}{i \hbar}\left[H_{\mathrm{osc}}-e E x, \rho_{11}(t)\right]+\Gamma_{L} e^{-x / \lambda} \rho_{00}(t) e^{-x / \lambda} \\
& -\frac{\Gamma_{R}}{2}\left(e^{2 x / \lambda} \rho_{11}(t)+\rho_{11}(t) e^{2 x / \lambda}\right)+\mathcal{L}_{\mathrm{damp}} \rho_{11}(t) .
\end{aligned}
$$

\section{CURRENT AND NOISE}

\section{A. Analytical development}

The current through the system is given by

$$
I^{\text {stat }}=e \Gamma_{L} \operatorname{Tr}_{\mathrm{osc}}\left(e^{-2 x / \lambda} \rho_{00}^{\text {stat }}\right)=e \Gamma_{R} \operatorname{Tr}_{\mathrm{osc}}\left(e^{2 x / \lambda} \rho_{11}^{\text {stat }}\right) \text {. }
$$

The trace is carried out over the oscillator basis and $\rho_{n n}^{\text {stat }}$ $=\lim _{t \rightarrow \infty} \rho_{n n}(t)$. To find the stationary solution, one needs to truncate the oscillator basis at some suitably large value $N$ (in practice, $N$ may reach 100 before convergence is achieved); since the resulting linear system has dimensions $2 N^{2} \times 2 N^{2}$ (single dot) or $10 N^{2} \times 10 N^{2}$ (triple dot), this becomes a critical issue. The method of solving this vast numerical task is commented on below.

Let us next consider the calculation of noise. In quantum optics one often resorts to a result known as the quantum regression theorem; this allows the calculation of any multi- 
time correlation function of system operators. Unfortunately, in NEMS this theorem can be applied only under very restricted circumstances: it can be used to calculate the noise (which is essentially the average of a product of current operators) within the three-dot device ${ }^{25}$ (because the current operators $\hat{I}_{L C}$ and $\hat{I}_{R C}$ operate within the system), while it is unapplicable for the single-dot case (and many other NEMS systems as well) because there the current operators involve operators belonging both to the baths (i.e., the electronic leads) and the system. A more general method is thus called for.

In order to compute the noise spectrum, we follow the ideas of Gurvitz and Prager, ${ }^{34}$ and introduce numberresolved density matrices $\rho_{i i}^{(n)}$, where $n$ is the number of electrons tunneled into the right lead by time $t$. Obviously, $\rho_{i i}(t)=\Sigma_{n} \rho_{i i}^{(n)}(t)$. The $\rho_{i i}^{(n)}$ obey

$$
\begin{aligned}
\dot{\rho}_{00}^{(n)}(t)= & \frac{1}{i \hbar}\left[H_{\mathrm{osc}}, \rho_{00}^{(n)}(t)\right]+\mathcal{L}_{\mathrm{damp}} \rho_{00}^{(n)}(t) \\
& -\frac{\Gamma_{L}}{2}\left\{e^{-2 x / \lambda}, \rho_{00}^{(n)}(t)\right\}+\Gamma_{R} e^{x / \lambda} \rho_{11}^{(n-1)}(t) e^{x / \lambda}, \\
\dot{\rho}_{11}^{(n)}(t)= & \frac{1}{i \hbar}\left[H_{\mathrm{osc}}-e E x, \rho_{11}^{(n)}(t)\right]+\mathcal{L}_{\mathrm{damp}} \rho_{11}^{(n)}(t) \\
& -\frac{\Gamma_{R}}{2}\left\{e^{2 x / \lambda}, \rho_{11}^{(n)}(t)\right\}+\Gamma_{L} e^{-x / \lambda} \rho_{00}^{(n)}(t) e^{-x / \lambda},
\end{aligned}
$$

with $\rho_{11}^{(-1)}(t) \equiv 0$. The mean current and the zero-frequency shot noise spectrum are given by ${ }^{35}$

$$
\begin{aligned}
& I=\left.e \frac{d}{d t} \sum_{n} n P_{n}(t)\right|_{t \rightarrow \infty}=\left.e \sum_{n} n \dot{P}_{n}(t)\right|_{t \rightarrow \infty}, \\
& S(0)=\left.2 e^{2} \frac{d}{d t}\left[\sum_{n} n^{2} P_{n}(t)-\left(\sum_{n} n P_{n}(t)\right)^{2}\right]\right|_{t \rightarrow \infty},
\end{aligned}
$$

where $P_{n}(t)=\operatorname{Tr}_{\mathrm{osc}}\left[\rho_{00}^{(n)}(t)+\rho_{11}^{(n)}(t)\right]$ are the probabilities of finding $n$ electrons in the right lead in time span 0 to $t$, i.e., precisely the objects needed for the FCS discussed below. We find $I=\sum_{n} n \dot{P}_{n}(t)=\Gamma_{R} \operatorname{Tr}_{\text {osc }}\left[e^{2 x / \lambda} \rho_{11}(t)\right]$, i.e., one recovers the stationary current found above. In a similar fashion, $\Sigma_{n} n^{2} \dot{P}_{n}(t)=\Gamma_{R} \operatorname{Tr}_{\text {osc }}\left\{e^{2 x / \lambda}\left[2 \Sigma_{n} n \rho_{11}^{(n)}(t)+\rho_{11}(t)\right]\right\}$, whose largetime asymptotics determines the shot noise according to (10). We have developed a generating function technique in Ref. 19 to extract this large-time limit. Here we skip the technical details; the upshot is that the zero-frequency noise, and thereby the Fano factor $F \equiv S(0) / 2 e I$, can be expressed in terms of the pseudoinverse of the Liouvillean (and the static limit of the density matrix known already from the current calculation):

$$
F=1-\frac{2 e \Gamma_{R}}{I} \operatorname{Tr}_{\mathrm{osc}}\left(e^{2 x / \lambda}\left[\mathcal{Q} \mathcal{L}^{-1} \mathcal{Q}\left(\begin{array}{c}
\Gamma_{R} e^{x / \lambda} \rho_{11}^{\text {stat }} e^{x / \lambda} \\
0
\end{array}\right)\right]_{11}\right) .
$$

Here $\mathcal{Q}$ is a projection operator that projects away from the stationary state for which the Liouvillean has the eigenvalue zero. The crucial point is that the pseudoinverse $\mathcal{R}$ of the
Liouvillean, defined as $\mathcal{Q} \mathcal{L}^{-1} \mathcal{Q} \equiv \mathcal{R}$, is tractable by similar numerical methods as used in the evaluation of the current. In our discussion of the full counting statistics given below, we analyze a toy model to illustrate some properties of the pseudoinverse $\mathcal{R}$.

\section{B. Comment on numerics: Some results}

As mentioned above, the superoperator structure of the Liouville equation leads to large matrices of the order of $N^{2} \times N^{2}$, where $N$ is the number of low-energy states kept in the calculation. A further complication arises from the fact that the stationary limit corresponds to the zero eigenvalue of the Liouvillean, $\mathcal{L} \rho=0$, forcing one to deal with singular matrices. The problems with the memory size can be circumvented by using iterative methods in which only $\mathcal{L} A$ for a given $A$ is needed ( $N \times N$ numbers), and one avoids the storage of the full $\mathcal{L}\left(N^{2} \times N^{2}\right.$ numbers). Using an iterative method raises the questions of convergence and the speed of convergence. We found that the so-called Arnoldi iteration technique was sufficient for our purposes (see Appendix A in Ref. 25), provided that one uses a suitable preconditioning. While there exists a substantial empirical body of knowledge of how to carry out the preconditioning, we are not aware of a complete algorithm. As an example, when solving for the pseudoinverse $\mathcal{R}$, we used the inverse of the "Sylvester part" $\mathcal{L}_{0}$ of $\mathcal{L}$ as preconditioner, ${ }^{26}$ finding the inverse of $\mathcal{L}_{0}$ is a relatively fast procedure. When calculating the noise (which amounts to solving an equation of the type $\mathcal{L} \mathbf{x}=\mathbf{b}$, where the vector $\mathbf{b}$ belongs in the range of $\mathcal{L}$ ), we used the generalized minimum residual method. ${ }^{36}$ Again, appropriate preconditioning was crucial.

Figure 2 shows a set of current vs damping curves for the single-dot shuttle. We draw attention to the following features. As damping is decreased, the current increases, approaching asymptotically the value $I=1 / 2 \pi \simeq 0.16$, i.e., one electron is transferred per cycle. In other words, this value of the current indicates that the shuttling transition has taken place, even in the quantum regime. The transition is not as sharp as found in the classical case. ${ }^{4}$ For large values of damping the current is much smaller and scales with the tunneling rate $\Gamma$. Very interestingly, we see a sharp increase in the current even without electric field $E=0$. Classically this does not happen, and therefore we interpret this crossover of being due to quantum shot noise.

We have, in several occasions, promoted the use of Wigner functions as an interpretative tool for the numerical results obtained for the stationary density matrix. The Wigner representation of the GME has also turned out to be a useful starting point for further analytic work. ${ }^{26,29,37}$ These phase-space representations have a simple form in the classical limit: the Wigner representation of a regularly moving harmonic oscillator is an ellipse. On the other hand, irregular motion under the influence of external noise gives rise to a Gaussian probability distribution centered at the origin. The charge resolved Wigner functions ( $n=0$ corresponds to an empty dot, while $n=1$ represents the occupied dot) are defined as 


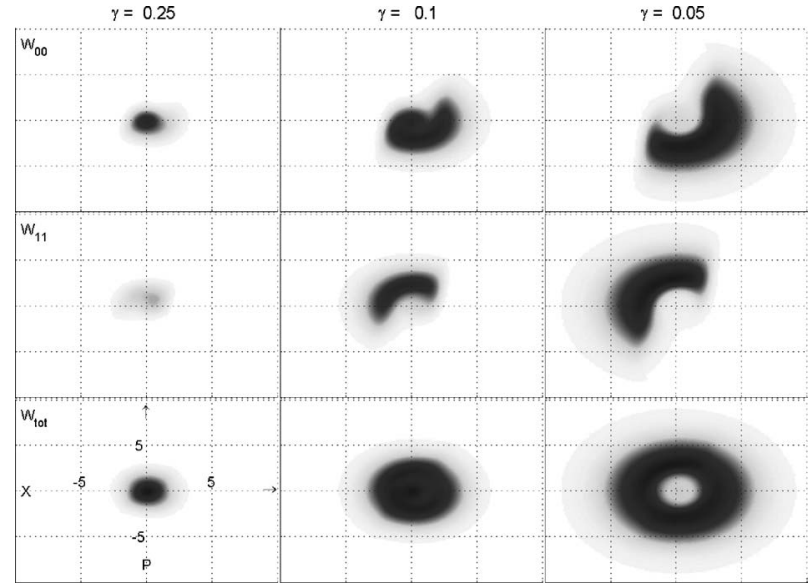

FIG. 3. Phase space picture of the tunneling-to-shuttling crossover. The respective rows show the Wigner distribution functions for the discharged $\left(W_{00}\right)$, charged $\left(W_{11}\right)$, and both $\left(W_{\text {tot }}\right)$ states of the oscillator in the phase space (horizontal axis-coordinate in units of $x_{0}=\sqrt{\hbar / m \omega}$, vertical axismomentum in $\left.\hbar / x_{0}\right)$. The values of the parameters are $\lambda=x_{0}, T=0, d$ $=0.5 x_{0}, \Gamma=0.05 \omega$. The values of $\gamma$ are in units of $\omega$. The Wigner functions are normalized within each column. (Reproduced from Ref. 23.)

$W_{n n}(X, P)=\int_{-\infty}^{\infty} \frac{d y}{2 \pi \hbar}\left\langle X-(y / 2)\left|\rho_{n n}^{\text {stat }}\right| X+(y / 2)\right\rangle \exp \left(i \frac{P y}{\hbar}\right)$,

and some representative results are given in Fig. 3. As expected, as damping is decreased, the fuzzy central spot evolves into a ring. The finite thickness of the ring is due to thermal noise, the randomness of the charge transport processes, and the position-momentum uncertainty. The fingerprints of shuttling are the asymmetric, banana-shaped areas observed for weakest damping: here one observes a strong correlation between the occupancy of the dot and the position and momentum. Thus, there is a large probability to have an occupied dot with a negative position coordinate and positive velocity (the dot has been filled in the neighborhood of the left contact), while there is a large probability of having an empty dot at positive $x$ values and negative velocity, i.e., on the return journey from the right contact. As the system approaches the classical limit, the thickness of the Wigner ring shrinks; this is illustrated in Fig. 4. As mentioned above, we expect that the noise of the quantum shuttle yields additional information about the nature of the charge transfer process. Figure 5 shows some of our numerical results. In the top panel, we see a relatively sharp crossover if

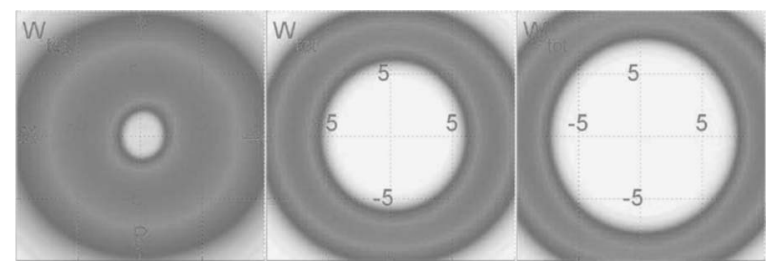

FIG. 4. Transition from quantum limit to classical limit. The "quantum thickness" of the Wigner ring shrinks and the radius increases, indicating larger maximal velocity and oscillation amplitude. The parameters are $\Gamma$ $=0.05 \omega, \lambda=x_{0}$ (left); $\Gamma=0.05 \omega, \lambda=2 x_{0}$ (middle); $\Gamma=0.01 \omega, \lambda=2 x_{0}$ (right).

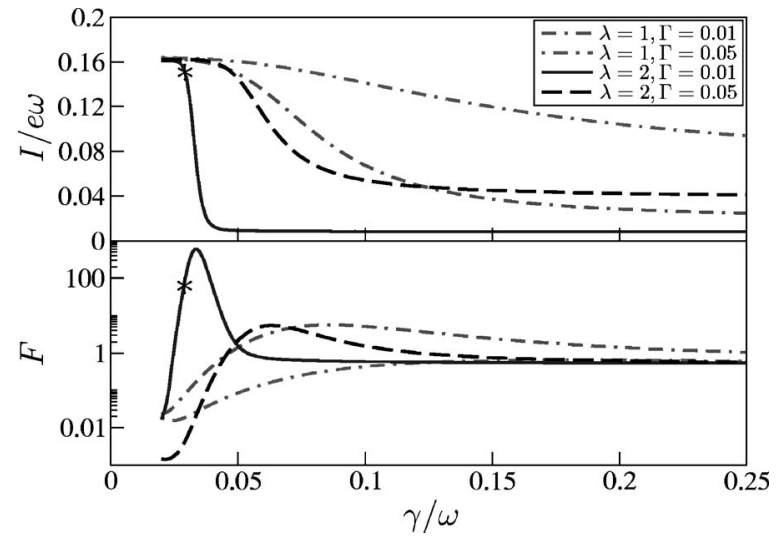

FIG. 5. Current $I$ and Fano factor $F$ vs damping $\gamma$. The $\gamma$ dependence of $I$ (upper panel) and $F$ on log scale (lower panel) for different transfer rates $\Gamma$ and tunneling lengths $\lambda$. The parameters are $\lambda=x_{0}, \Gamma=0.01 \omega$ (full); $\lambda=x_{0}$, $\Gamma=0.05 \omega$ (long dashes); $\lambda=2 x_{0}, \quad \Gamma=0.01 \omega$ (short dashes); $\lambda=2 x_{0}, \quad \Gamma$ $=0.05 \omega$ (dots) with $x_{0}=\sqrt{\hbar / m \omega}$. Other parameters are $e E / m \omega^{2}=0.5 x_{0}$ and $T=0$. The current is in units of $e \omega$ while $\gamma$ in units of $\omega$. The asterisk defines the parameters of Wigner distributions in Fig. 6. (Reproduced from Ref. 19.)

the tunneling length is larger than the length scale $x_{0}$ $=\sqrt{\hbar /(m \omega)}$, i.e., as one approaches the classical limit. In the bottom panel we show the Fano factor for the same parameters as in the top panel. One should note that a logarithmic scale is used; thus, one observes (i) a huge enhancement at the tunneling crossover, in particular, for parameters approaching the semiclassical limit and (ii) tiny Fano factors below the shuttling crossover. Thus, even well in the quantum regime shuttling is a highly ordered charge transfer process.

Figure 6 shows the phase-space plot for the parameter values indicated by the asterisk in Fig. 5. The interpretation is very suggestive: for these parameters tunneling and shuttling coexist. Our numerics thus confirm the suggestion put forward in the analytical study of Ref. 29. The large value of the Fano factor can be understood as a slow switching process between the two possible current channels (tunneling and shuttling). In the following section we elaborate this point further.
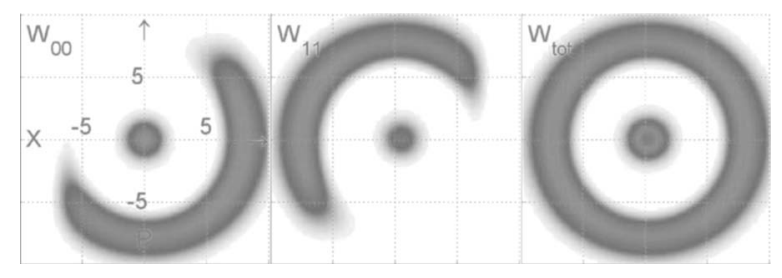

FIG. 6. Phase space picture of the shuttle around the transition where the shuttling and tunneling regimes coexist. The respective rows show the Wigner distribution functions for the discharged $\left(W_{00}\right)$, charged $\left(W_{11}\right)$, and both $\left(W_{\mathrm{tot}}=W_{00}+W_{11}\right)$ states of the oscillator in the phase space (horizontal axis-coordinate in units of $x_{0}=\sqrt{\hbar / m \omega}$, vertical axis-momentum in $\hbar / x_{0}$ ). The values of the parameters are $\lambda=2 x_{0}, e E / m \omega^{2}=0.5 x_{0}, \gamma=0.029 \omega, \Gamma$ $=0.01 \omega$, and $T=0$. 


\section{FULL COUNTING STATISTICS}

\section{A. Calculational procedure}

We begin by introducing some notation. We recall that the Liouvillean, which is a non-Hermitian operator, has a single eigenvalue equal to zero with $\hat{\rho}^{\text {stat }}$ being the corresponding (normalized and unique) right eigenvector, we denote this eigenvector by $|0\rangle\rangle$. The corresponding left eigenvector is the identity operator $\hat{1}$ which we denote by $\langle\langle\widetilde{0}|$, and we have $\langle\langle\widetilde{0} \mid 0\rangle\rangle \equiv \operatorname{Tr}\left(\hat{1}^{\dagger} \hat{\rho}^{\text {stat }}\right)=1$. The pair of eigenvectors allows us to define the complementary projectors $\mathcal{P} \equiv|0\rangle\rangle\langle\langle\widetilde{0}|$ and $\mathcal{Q} \equiv 1-\mathcal{P}$ obeying the relations $\mathcal{P} \mathcal{L}=\mathcal{L} \mathcal{P}=0$ and $\mathcal{Q} \mathcal{L}$ $=\mathcal{L}$. We will also need the pseudoinverse of the Liouvillean $\mathcal{R} \equiv \mathcal{Q} \mathcal{L}^{-1} \mathcal{Q}$, which is well defined, since the inversion is performed only in the subspace spanned by $\mathcal{Q}$, where $\mathcal{L}$ is regular. Rather than attempting to calculate the complete probability distribution $P_{n}(t)=\operatorname{Tr}\left[\hat{\rho}^{(\mathrm{n})}(\mathrm{t})\right]$ directly, it turns out to be easier to evaluate the cumulant generating function $S(t, \chi)$ :

$$
e^{S(t, \chi)}=\sum_{n=-\infty}^{\infty} P_{n}(t) e^{i n \chi}
$$

From $S(t, \chi)$ we find the $m$ th cumulant of the charge distribution by taking the $m$ th derivative with respect to the counting field $\chi$ at $\chi=0$,

$$
\left\langle\left\langle n^{m}\right\rangle\right\rangle(t)=\left.\frac{\partial^{n} S}{\partial(i \chi)^{m}}\right|_{\chi=0},
$$

and from the knowledge of all cumulants we can reconstruct $P_{n}(t)$. The cumulants of the current in the stationary limit $t \rightarrow \infty$ are given by the time derivative of the charge cumulants:

$$
\left\langle\left\langle I^{m}\right\rangle\right\rangle=\left.\frac{d}{d t}\left\langle\left\langle n^{m}\right\rangle\right\rangle(t)\right|_{t \rightarrow \infty} .
$$

The first two current cumulants give the average current running through the system and the zero-frequency current noise, respectively. We have recently developed a systematic perturbation theory to calculate the higher cumulants, ${ }^{22}$ and we just quote the final results

$$
\begin{aligned}
\langle\langle I\rangle\rangle= & \langle\langle\widetilde{0}|\mathcal{I}| 0\rangle\rangle, \\
\left\langle\left\langle I^{2}\right\rangle\right\rangle= & \langle\langle\widetilde{0}|\mathcal{J}| 0\rangle\rangle-2\langle\langle\widetilde{0}|\mathcal{I} \mathcal{R} \mathcal{I}| 0\rangle\rangle, \\
\left\langle\left\langle I^{3}\right\rangle\right\rangle= & \langle\langle\widetilde{0}|\mathcal{I}| 0\rangle\rangle-3\langle\langle\widetilde{0}|\mathcal{I} \mathcal{R} \mathcal{J}+\mathcal{J} \mathcal{R} \mathcal{I}| 0\rangle\rangle \\
& -6\langle\langle\widetilde{0}|\mathcal{I} \mathcal{R}(\mathcal{R} \mathcal{I} \mathcal{P}-\mathcal{I} \mathcal{I}) \mathcal{I}| 0\rangle\rangle,
\end{aligned}
$$

where

$$
\begin{aligned}
& \mathcal{I}=\mathcal{I}^{+}-\mathcal{I}^{-}, \\
& \mathcal{J}=\mathcal{I}^{+}+\mathcal{I}^{-} .
\end{aligned}
$$

The explicit expressions for the current superoperators $\mathcal{I}^{ \pm}$ depend on the problem at hand; two examples are given in Ref. 22 (see also the analytic toy model discussed below).
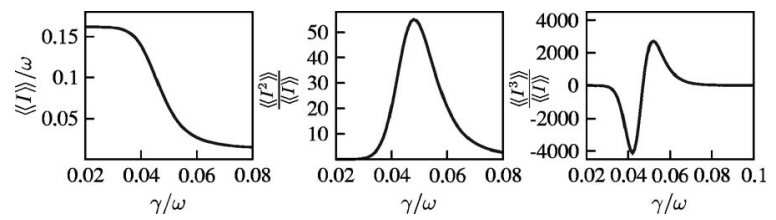

FIG. 7. The first three cumulants for the one-dot shuttle as a function of the damping $\gamma$. The parameters are $\lambda=1.5 x_{0}$ and $d=e E / m \omega^{2}=0.5 x_{0}$.

We emphasize the utility of these formulas: they imply that once the hard numerical problems with the stationary case are solved, the higher cumulants are essentially immediately accessible. Using the systematic perturbation theory developed in Ref. 22 algebraic formulas for the fourth, fifth, etc., cumulant can be generated with computer routines performing symbolic manipulations. In Fig. 7, we show numerical results for the first three cumulants for the single-dot shuttle.

\section{B. Toy model}

The general formulation for calculation of higher cumulants given above is very practical also in analytic calculations. Here we illustrate it by considering a toy model, for which the current and noise are well known, while the third cumulant cannot be considered as a standard result. Specifically, we consider a two-level system, whose occupation probabilities obey the master equation $\left(\mathbf{p}=\left(\begin{array}{ll}p_{0} & p_{1}\end{array}\right)^{\mathrm{T}}\right)$

$$
\dot{\mathbf{p}}=\mathcal{L} \mathbf{p}=\left(\begin{array}{cc}
-\Gamma_{L} & \Gamma_{R} \\
\Gamma_{L} & -\Gamma_{R}
\end{array}\right) \mathbf{p}
$$

The relevance of this model to the shuttling dynamics is based on the fact that in the coexistence regime there are two "states": the incoherent tunneling current channel (reflected by the central spot in the Wigner function description) and the orderly shuttling channel (reflected) by the ring, and that the system can be visualized as fluctuating between these two states. The bare two-level system, as discussed in this section, needs a slight modification to take into account that in the coexistence regime one deals with current channels and not states, and further discussion can be found in Ref. 22, see also Ref. 38 for a related model. The Liouvillean of Eq. (19) has the following right and left null vectors:

$$
|0\rangle\rangle=\frac{1}{\Gamma_{L}+\Gamma_{R}}\left(\begin{array}{c}
\Gamma_{R} \\
\Gamma_{L}
\end{array}\right), \quad\left\langle\langle\widetilde{0}|=\left(\begin{array}{ll}
1 & 1
\end{array}\right),\right.
$$

which satisfy $\mathcal{L}|0\rangle\rangle=0=\langle\langle\widetilde{0}| \mathcal{L}$ and $\langle\langle\widetilde{0} \mid 0\rangle\rangle=1$, as is readily verified. We can immediately form the projectors $\mathcal{P}$ and $\mathcal{Q}$ :

$$
\begin{aligned}
& \mathcal{P}=|0\rangle\rangle\left\langle\langle\widetilde{0}|=\frac{1}{\Gamma_{R}+\Gamma_{L}}\left(\begin{array}{cc}
\Gamma_{R} & \Gamma_{R} \\
\Gamma_{L} & \Gamma_{L}
\end{array}\right),\right. \\
& \mathcal{Q}=1-\mathcal{P}=\frac{1}{\Gamma_{R}+\Gamma_{L}}\left(\begin{array}{cc}
\Gamma_{L} & -\Gamma_{R} \\
-\Gamma_{L} & \Gamma_{R}
\end{array}\right) .
\end{aligned}
$$

We also record the current superoperator 


$$
\mathcal{I}_{0 R}=\left(\begin{array}{cc}
0 & \Gamma_{R} \\
0 & 0
\end{array}\right)
$$

Next, we need the eigenvector $|\nu\rangle\rangle$ of $\mathcal{L}$ which does not belong to the null space (here, the situation is quite simple because the full problem is two dimensional with a one dimensional null space), and the associated eigenvalue $\lambda_{\nu}$. We readily find

$$
\begin{aligned}
& \left.\lambda_{\nu}=-\left(\Gamma_{R}+\Gamma_{L}\right), \quad|\nu\rangle\right\rangle=\left(\begin{array}{c}
1 \\
-1
\end{array}\right), \\
& \left\langle\left\langle\widetilde{\nu}=\frac{1}{\Gamma_{R}+\Gamma_{L}}\left(\Gamma_{L}-\Gamma_{R}\right),\right.\right.
\end{aligned}
$$

and can thus compute the pseudoinverse $\mathcal{R}$,

$$
\begin{aligned}
\mathcal{R} & \left.=\mathcal{Q} \mathcal{L}^{-1} \mathcal{Q}=\frac{1}{\lambda_{\nu}}|\nu\rangle\right\rangle\left\langle\langle\widetilde{\nu}|=-\frac{1}{\left(\Gamma_{R}+\Gamma_{L}\right)^{2}}\left(\begin{array}{cc}
\Gamma_{L} & -\Gamma_{R} \\
-\Gamma_{L} & \Gamma_{R}
\end{array}\right)\right. \\
& =\frac{1}{\lambda_{\nu}} \mathcal{Q} .
\end{aligned}
$$

With these results at hand, the evaluation of various cumulants is reduced to simple matrix multiplications, and we quote the results

$$
\begin{aligned}
& \langle\langle\mathcal{I}\rangle\rangle=\frac{\Gamma_{L} \Gamma_{R}}{\Gamma_{L}+\Gamma_{R}}, \\
& \left\langle\left\langle\mathcal{I}^{2}\right\rangle\right\rangle=\frac{\Gamma_{R}^{2}+\Gamma_{L}^{2}}{\left(\Gamma_{R}+\Gamma_{L}\right)^{2}}\langle\langle\mathcal{I}\rangle\rangle, \\
& \left\langle\left\langle\mathcal{I}^{3}\right\rangle\right\rangle=\frac{\Gamma_{R}^{4}-2 \Gamma_{R}^{3} \Gamma_{L}+6 \Gamma_{R}^{2} \Gamma_{L}^{2}-2 \Gamma_{R} \Gamma_{L}^{3}+\Gamma_{L}^{4}}{\left(\Gamma_{R}+\Gamma_{L}\right)^{2}}\langle\langle\mathcal{I}\rangle\rangle .
\end{aligned}
$$

These results have been reported in the literature (see, e.g., Refs. 12 and 39; however, the derivation presented here is quite different. We believe that the above method of calculation can be very useful in extending several previous results, such as those obtained in by Kießlich et al. ${ }^{40}$

\section{Experimental status}

To summarize the experimental status very shortly, we do not believe that the shuttling transition has been observed so far. Having stated this negative conclusion, we hasten to point out that we think that the experimental situation is very promising, and that a crucial experiment could be just behind the corner.

Two key experiments have been reported, which contain several important ingredients. In the experiment of Park et al. ${ }^{5}$ a $\mathrm{C}_{60}$ molecule was placed in a break junction, and the current-voltage characteristics showed clear indications of effects due to vibrational quanta. We believe that the observed values of the current are too small to be attributed to shuttling (recall that the shuttling current has the universal value of $1 / 2 \pi$ ), and that the system is in the tunneling limit. In the experiment of Erbe et al. ${ }^{41}$ the system was driven: indeed electrons were shuttled, but the experiment was not designed to observe the shuttling transition as a function of a control parameter. Our optimism is based on a number of new structures that are currently being explored in the literature, and in particular we find the structures of Scheible and Blick, ${ }^{42}$ where a soft silicon pillar forms the movable part, very promising. We also believe that the theoretical methods outlined above are suitable for modeling quantitatively the forthcoming experiments.

\section{CONCLUSION}

We have discussed at length some properties of a nanomechanical device, the quantum shuttle, which we believe can be an important component in future applications, for example, measurements of very small displacements. While the quantitative results of the present work apply to very specific and strongly idealized models, we believe that many of the phenomena we address are generic, and will be observed in near future. One of the central messages we want to pass is that the fluctuation properties of these devices contain a wealth of information, and that this information may be essential in identifying the key charge transfer processes in these devices.

${ }^{1}$ H. G. Craighead, "Nanoelectromechanical systems," Science 290, 1532 (2000).

${ }^{2}$ A. N. Cleland, Foundations of Nanomechanics, Advanced Texts in Physics (Springer, Berlin, 2003).

${ }^{3}$ M. Blencowe, "Quantum electromechanical systems," Phys. Rep. 395, 159 (2004).

${ }^{4}$ L. Y. Gorelik, A. Isacsson, M. V. Voinova, B. Kasemo, R. I. Shekhter, and M. Jonson, "Shuttle mechanism for charge transfer in Coulomb blockade nanostructures," Phys. Rev. Lett. 80, 4526 (1998).

${ }^{5}$ H. Park, J. Park, A. K. L. Lim, E. H. Anderson, A. P. Alivisatos, and P. L. McEuen, "Nanomechanical oscillations in a single- $\mathrm{C}_{60}$ transistor," Nature (London) 407, 57 (2000).

${ }^{6} \mathrm{D}$. Boese and H. Schoeller, "Influence of nanomechanical properties on single-electron tunneling: A vibrating single-electron transistor," Europhys. Lett. 54, 668 (2001).

${ }^{7}$ K. D. McCarthy, N. Prokof'ev, and M. T. Tuominen, "Incoherent dynamics of vibrating single-molecule transistors," Phys. Rev. B 67, 245415 (2003).

${ }^{8} \mathrm{~K}$. Flensberg, "Tunneling broadening of vibrational sidebands in molecular transistors," Phys. Rev. B 68, 205323 (2003).

${ }^{9} \mathrm{~S}$. Braig and K. Flensberg, "Vibrational sidebands and dissipative tunneling in molecular transistors," Phys. Rev. B 68, 205324 (2003).

${ }^{10}$ D. Fedorets, L. Y. Gorelik, R. I. Shekhter, and M. Jonson, "Vibrational instability due to coherent tunneling of electrons," Europhys. Lett. 58, 99 (2002).

${ }^{11}$ Sh. Kogan, Electronic Noise and Fluctuations in Solids (Cambridge University Press, Cambridge, 1996).

${ }^{12}$ Ya. M. Blanter and M. Büttiker, "Shot noise in mesoscopic conductors," Phys. Rep. 336, 1 (2000).

${ }^{13}$ C. Beenakker and C. Schönenberger, "Quantum shot noise," Phys. Today 56(5), 37 (2003).

${ }^{14}$ A. Mitra, I. Aleiner, and A. J. Millis, "Phonon effects in molecular transistors: Quantum and classical treatment," Phys. Rev. B 69, 245302 (2004).

${ }^{15}$ F. Pistolesi, "Full counting statistics of a charge shuttle," Phys. Rev. B 69, 245409 (2004)

${ }^{16}$ A. D. Armour, "Current noise of a single-electron transistor coupled to a nano-mechanical resonator," Phys. Rev. B 70, 165315 (2004).

${ }^{17}$ N. M. Chtchelkatchev, W. Belzig, and C. Bruder, "Charge transport through a set with a mechanically oscillating island," Phys. Rev. B 70, 193305 (2004).

${ }^{18} \mathrm{~A}$. Isacsson and T. Nord, "Low-frequency current noise of the singleelectron shuttle," Europhys. Lett. 66, 708 (2004).

${ }^{19}$ T. Novotný, A. Donarini, C. Flindt, and A.-P. Jauho, "Shot noise of a quantum shuttle," Phys. Rev. Lett. 92, 248302 (2004).

${ }^{20}$ Ya. M. Blanter, O. Usmani, and Yu. V. Nazarov, "Single-electron tunnel- 
ing with strong mechanical feedback," Phys. Rev. Lett. 93, 136802 (2004).

${ }^{21}$ B. Reulet, J. Senzier, and D. E. Prober, "Environmental effects in the third moment of voltage fluctuations in a tunnel junction," Phys. Rev. Lett. 91, 196601 (2003).

${ }^{22}$ C. Flindt, T. Novotný, and A.-P. Jauho, "Full counting statistics of nanoelectromechanical systems," Europhys. Lett. 69, 475 (2005).

${ }^{23}$ T. Novotný, A. Donarini, and A.-P. Jauho, "Quantum shuttle in phase space," Phys. Rev. Lett. 90, 256801 (2003).

${ }^{24}$ A. Donarini, T. Novotný, and A.-P. Jauho, "Quantum theory of shuttling instability in a movable quantum dot array," Semicond. Sci. Technol. 19, S430 (2004).

${ }^{25}$ C. Flindt, T. Novotný, and A.-P. Jauho, "Current noise in a vibrating quantum dot array," Phys. Rev. B 70, 205334 (2004).

${ }^{26}$ A. Donarini, "Dynamics of shuttle devices," Ph.D. thesis, MIC, Technical University of Denmark, 2004.

${ }^{27}$ R. I. Shekhter, Y. Galperin, L. Y. Gorelik, A. Isacsson, and M. Jonson, "Shuttling of electrons and Cooper pairs," J. Phys.: Condens. Matter 15, R441 (2003).

${ }^{28}$ D. Fedorets, "Quantum description of shuttle instability in a nanoelectromechanical single-electron transistor," Phys. Rev. B 68, 033106 (2003).

${ }^{29}$ D. Fedorets, L. Y. Gorelik, R. I. Shekhter, and M. Jonson, "Quantum shuttle phenomena in a nanoelectromechanical single-electron transistor," Phys. Rev. Lett. 92, 166801 (2004)

${ }^{30}$ A. Yu. Smirnov, L. G. Mourokh, and N. J. M. Horing, "Temperature dependence of electron transport through a quantum shuttle," Phys. Rev. B
69, 155310 (2004).

${ }^{31}$ A. D. Armour and A. MacKinnon, "Transport via a quantum shuttle," Phys. Rev. B 66, 035333 (2002).

${ }^{32}$ C. W. Gardiner and P. Zoller, Quantum Noise, 2nd ed. (Springer, New York, 2000).

${ }^{33}$ U. Weiss, Quantum Dissipative Systems, Series in Modern Condensed Matter Physics Vol. 10 (World Scientific, Singapore, 1999).

${ }^{34}$ S. A. Gurvitz and Ya. S. Prager, "Microscopic derivation of rate equations for quantum transport," Phys. Rev. B 53, 15932 (1996).

${ }^{35}$ B. Elattari and S. A. Gurvitz, "Shot noise in coupled dots and the fractional charges," Phys. Lett. A 292, 289 (2002).

${ }^{36}$ C. Flindt, "Current noise in a 3-dot quantum shuttle," Master's thesis, MIC, Technical University of Denmark, 2004.

${ }^{37}$ D. Fedorets, L. Y. Gorelik, R. I. Shekhter, and M. Jonson, "Spintronics of a nanoelectromechanical shuttle,"( cond-mat/0408591).

${ }^{38}$ A. N. Jordan and E. V. Sukhorukov, "Transport statistics of bistable systems," Phys. Rev. Lett. 93, 260604 (2004).

${ }^{39}$ M. J. M. de Jong, "Distribution of transmitted charge through a doublebarrier junction,” Phys. Rev. B 54, 8144 (1996).

${ }^{40}$ G. Kießlich, A. Wacker, and E. Schöll, "Shot noise of coupled semiconductor quantum dots," Phys. Rev. B 68, 125320 (2003).

${ }^{41}$ A. Erbe, C. Weiss, W. Zwerger, and R. H. Blick, "Nanomechanical resonator shuttling single electrons at radio frequencies," Phys. Rev. Lett. 87, 096106 (2001).

${ }^{42}$ D. C. Scheible and R. H. Blick, "Silicon nanopillars for mechanical single electron transport," Appl. Phys. Lett. 84, 4632 (2004). 